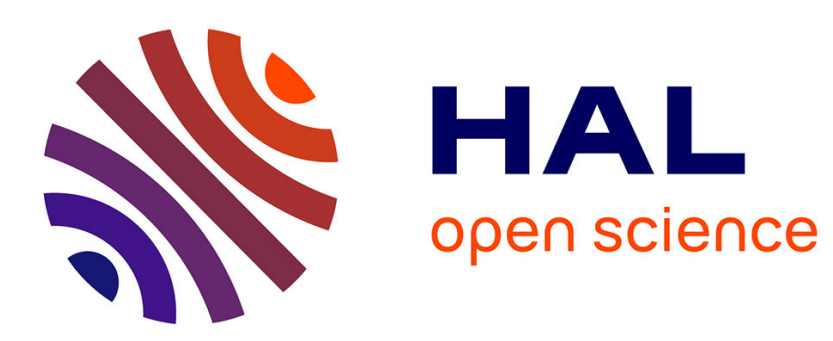

\title{
Architecture dominicaine et promotion de nouveaux saints : autour de la tombe de Clément IV à Santa Maria in Gradi (Viterbe)
}

Haude Morvan

\section{- To cite this version:}

Haude Morvan. Architecture dominicaine et promotion de nouveaux saints: autour de la tombe de Clément IV à Santa Maria in Gradi (Viterbe). Bulletin Monumental, 2013, 171 (2), pp.99-106. hal-01792414

\author{
HAL Id: hal-01792414 \\ https://hal.science/hal-01792414
}

Submitted on 15 Oct 2019

HAL is a multi-disciplinary open access archive for the deposit and dissemination of scientific research documents, whether they are published or not. The documents may come from teaching and research institutions in France or abroad, or from public or private research centers.
L'archive ouverte pluridisciplinaire $\mathbf{H A L}$, est destinée au dépôt et à la diffusion de documents scientifiques de niveau recherche, publiés ou non, émanant des établissements d'enseignement et de recherche français ou étrangers, des laboratoires publics ou privés. 


\section{b u 1 l e t i n m o n u m n t a 1}




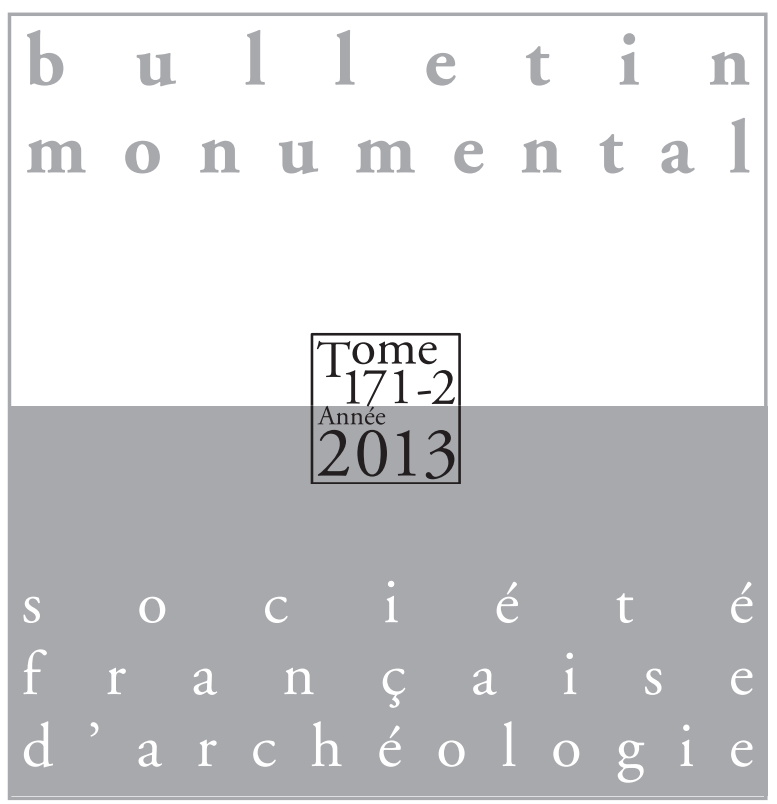

Revue publiée avec le soutien du Centre National du Livre 
Toute reproduction de cet ouvrage, autre que celles prévues à l'article L. 122-5 du Code de la propriété intellectuelle, est interdite, sans autorisation expresse de la Société française d'archéologie et duldes auteur(s) des articles et images d'illustration concernés. Toute reproduction illégale porte atteinte aux droits duldes auteurs(s) des articles, à ceux des auteurs ou des institutions de conservation des images d'illustration, non tombées dans le domaine public, pour lesquelles des droits spécifiques de reproduction ont été négociés, enfin à ceux de l'éditeur-diffuseur des publications de la Société française d'archéologie.

(C) Société Française d'Archéologie

Siège social : Cité de l'Architecture et du Patrimoine, 1, place du Trocadéro et du 11 Novembre, 75116 Paris. Bureaux : 5, rue Quinault, 75015 Paris, tél. : 01427308 07, mail : sfa.sfa@wanadoo.fr

Revue trimestrielle, t. 171-II, juin 2013

ISSN : 0007-4730

CPPAP : 0112 G 86537

ISBN : 978-2-901837-45-9

Diffusion : Éditions A. \& J. Picard, 82, rue Bonaparte, 75006 Paris

Tél. librairie 0143269673 - Fax 0143264264

achats@librairie-picard.com

www.librairie-picard.com 


\section{TABLE DES MATIËRES}

\section{ARTICLES}

Architecture dominicaine et promotion de nouveaux saints : autour de la tombe de Clément IV à Santa Maria in Gradi (Viterbe), par Haude Morvan.

Le cycle peint du château de Cruet (Savoie, vers 1307) : une représentation du roman de Girart de Vienne? par Térence Le Deschault de Monredon.

L'abbaye de moniales cisterciennes de Clairefontaine (Luxembourg). Synthèse archéo-historique des quatre phases de construction, XIII-XVII' siècle, par Davy Herremans et Thomas Coomans.

\section{MÉLANGeS}

Entre art et technique : les innovations "à la française " d'un fervent visiteur de l'Italie, Geoffroy Tory. À propos d'une exposition du Musée de la Renaissance d'Écouen, par Marie Madeleine Fontaine.

LIBRE-PROPOS

Fontainebleau avant 1541. Observations sur l'article de Thomas Clouet, par Françoise Boudon et Jean Guillaume.....

\section{ACTUALité}

Doubs. Besançon. État des études sur les caves médiévales (XII'-XV siècle) [Jean-Denis Clabaut]

Loiret. Orléans. Étude d'une maison en pan de bois de la fin du XIV siècle, 25, rue de la Poterne (Clément Alix).

Haute-Vienne. Limoges. Découverte d'un mausolée de l'Antiquité tardive au sein de la nécropole de Saint-Martial, 1, rue de la Courtine (Xavier Lhermite).

ChroniQue

Époque médiévale. Architecture civile et religieuse. Topographie ecclésiale du haut Moyen Âge dans le sud-ouest de la Grande-Bretagne (Élisabeth Lorans). - Boves (Somme) : étude du mobilier archéologique et des marques de calibrage lapidaire (Catherine Brut). — Église Saint-Laurent de Beaulieu-lès-Loches (Indre-et-Loire) [Yves Blomme]. — Signalement : entre Strasbourg et Orléans, retour sur le « dessin 21 » de l'Euvre Notre-Dame (Benoît Jordan).

Architecture castrale. Le château de Sanzay (Deux-Sèvres) : nouvelle datation (Dominique Hervier)

Histoire urbaine. Pontoise au Moyen Âge : méthodes d'étude de la ville ordinaire (Ezéchiel Jean-Courret).

Histoire du costume. Ornementations métalliques du vêtement médiéval : deux sources inédites concernant les ceintures (Nadège Gauffre Fayolle).

Époque classique. Architecture civile et religieuse. L'urbanisme parisien et le mécénat artistique de Marie de Médicis (Ronan Bouttier). - L'évolution du statut de l'architecture sous le règne de Louis XIV: le rôle de Charles Le Brun (Marianne Cojannot-Le Blanc). — Le projet de Jadot pour la Hofburg (Bertrand Jestaz).....

Architecture XIX et $\mathrm{XX}^{\mathrm{e}}$ siècle. Signalement : un avant-gardiste de l'architecture néo-renaissance (Dominique Hervier). - Repenser l'architecture religieuse en Belgique au XX' siècle (Alain Nafilyan).

BiBLIOGRAPHIE

Cluny. Neil Stratford (dir.), Cluny 910-2010. Onze siècles de rayonnement; Neil Stratford (dir.), Corpus de la sculpture de Cluny. Tome 1. Les parties orientales de la Grande Église Cluny III; Nicolas Reveyron (dir.) avec la collab. de Gilles Rollier, Hugues de Semur (1024-1109). Lumières clunisiennes (Christian Gensbeitel)

Architecture. Frédéric Boutoulle, Dany Barraud et Jean-Luc Piat (éd.), Fabrique d'une ville médiévale. Saint-Émilion au Moyen Âge (Anne-Laure Napoléone). — Anne Baud (dir.), Espace ecclésial et liturgie au Moyen Âge (Yann Codou). — Ewa Luziniecka, Zygmunt Swiechowski et Robert Kunkel, Architektura opactw cysterskich. Maloposkie filie Morimond / The architecture of 
cistercian Abbeys. Morimond filiations in Little Poland (Éliane Vergnolle). — Monique Chatenet, Krista De Jonge, Ethan Matt Kavaler et Norbert Nußbaum (dir.), Le Gothique de la Renaissance (Étienne Faisant). — Mathieu Lours, L'autre temps des cathédrales, du concile de Trente à la Révolution française (Stéphanie Diane Daussy). — Nicolas Courtin, L'art d'habiter à Paris au XVII siècle : l'ameublement des hôtels particuliers (Mark Girouard)

Ingénieurs et architectes. Philippe Bragard, Dictionnaire biographique des ingénieurs des fortifications. Pays-Bas espagnols, principauté de Liège, Franche-Comté, 1504-1713 (David Buisseret). - Robin Middleton et Marie-Nöelle Baudouin-Matuszek, Jean Rondelet: The Architect as Technician (Patrick Ponsot). — Jean-Yves Andrieux, (dir.), Arthur Regnault architecte (1839-1932): la quintessence de l'art sacré (Antoine Le Bas)

Vitraux. Karine Boulanger, Les vitraux de la cathédrale d'Angers (Christine Hediger). - Bruno Togni et al., Carrelages et dallages du XII au XIX siècle (Martine Diot).

RÉSUMÉS ANALYTIQUES.

LISTE DES AUTEURS

Achevé d'imprimer sur les presses de l'imprimerie de Montligeon

à Saint-Hilaire-le-Châtel en juin 2013

$\mathrm{N}^{\circ}$ d'impression : 26001

Dépôt-légal : juin 2013 


\title{
ARCHITECTURE DOMINICAINE ET PROMOTION DE NOUVEAUX SAINTS : AUTOUR DE LA TOMBE DE Clément IV à Santa Maria in Gradi (Viterbe)
}

\author{
Haude MORVAN *
}

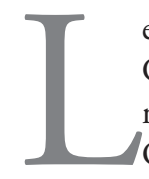

e monument funéraire du pape Clément IV († 1268), initialement situé à Santa Maria in Gradi, église dominicaine de Viterbe, est notable à bien des égards. Il marque une étape importante dans l'histoire de la sculpture italienne et illustre la créativité et l'innovation du milieu dominicain en matière artistique. À ce titre, il a été l'objet de nombreuses études.

La reconstitution des vicissitudes du monument à travers une récolte méthodique de la documentation a été menée en particulier par Anna Maria D’Achille ${ }^{1}$. L'histoire du tombeau pose des problèmes dès son installation. Entre 1268 et 1276 , le corps fut accaparé par les chanoines de la cathédrale de Viterbe, malgré les bulles répétées enjoignant de rendre la dépouille aux frères prêcheurs chez qui Clément IV avait élu sépulture. Certaines bulles parlent déjà d'un sépulcre, mais il est impossible de savoir s'il s'agit vraiment de celui qui fut déplacé ensuite chez les Dominicains. De fait, le doute demeure sur la datation du tombeau, entre la mort du pape en 1268 et la restitution du corps aux Dominicains en 1276. Par la suite, plusieurs évènements impliquèrent d'importantes destructions et restaurations. En 1741, alors que l'église était en cours de reconstruction, la tombe de Clément IV fut déplacée dans la chapelle Saint-Dominique (à gauche de l'abside principale). C'est alors que fut perdue la fresque qui représentait sainte Edwige (canonisée par Clément IV) à genoux devant la Vierge. Puis, en 1885, suite à la suppression des congrégations religieuses, le couvent dominicain fut transformé en prison et le monument funéraire du pape à nouveau démonté pour être transféré dans l'église franciscaine de Viterbe où il se trouve encore aujourd'hui (fig. 1). De lourdes restaurations furent faites à ce moment, notamment sur le baldaquin. En 1944, le bombardement qui détruisit une partie de la ville toucha l'église franciscaine. Les photos prises après le drame montrent le piètre état dans lequel fut réduit le tombeau de Clément IV. Son état actuel est le fruit de la restauration de 1949. Au terme de toutes ces péripéties, les seules parties authentiques du monument sont le gisant et le sarcophage, remploi d'une pièce antique. Ce dernier n'est toutefois pas dans sa position d'origine : on voit aujourd'hui la face strigillée, primitivement tournée vers le mur, puisque la face ornée de motifs cosmatesques conçue au XIII siècle pour être visible a été lourdement endommagée lors du bombardement.

L'étude du tombeau de Clément IV touche plusieurs thématiques cruciales de l'histoire de l'art funéraire italien. Peter Cornelius Claussen s'est interrogé sur la formation du sculpteur, Pietro di Oderisio ${ }^{2}$. Julian Gardner a développé la question de l'influence française, particulièrement convaincante en ce qui concerne le gisant et le baldaquin gothique ${ }^{3}$. En effet, il est tout à fait significatif que le gisant, forme courante en France, en Angleterre et en Allemagne à partir du début du XIII siècle, apparaisse en Italie avec la tombe d'un pape français. L'absence d'antécédent italien à la typologie introduite par le sépulcre de Clément IV fait toutefois débat. Ingo Herklotz s'est attaché à replacer celui-ci dans l'histoire de la tombe murale et de la représentation du défunt en contexte funéraire en Italie depuis l'époque paléochrétienne ${ }^{4}$. Plusieurs arguments ont été soulevés pour nuancer la primauté italienne du gisant de Clément IV, notamment la quantité non mesurable de monuments funéraires perdus ou encore la difficulté d'attribuer au sépulcre du pontife une date précise entre 1268 et 1276 . Ce débat a notamment été alimenté par une esquisse sommaire du XVIII ${ }^{\mathrm{e}}$ siècle représentant le tombeau de l'évêque Paolo di Pafo, mort en 1268, six mois avant Clément IV, et inhumé lui aussi à Santa Maria in Gradi ${ }^{5}$. La représentation vague d'une silhouette couchée a conduit plusieurs auteurs à s'interroger sur la possible existence d'un gisant ${ }^{6}$. En 2000, Silvia Pacella a clos définitivement la question en publiant un autre dessin du monument de Paolo di Pafo, découvert dans un manuscrit de la Bibliothèque nationale de Florence (ms. N.A.1216), attribué par la chercheuse à Feliciano Bussi ${ }^{7}$. Grâce à ce nouveau dessin, il apparaît clairement que l'effigie n'était pas un gisant en ronde-bosse mais était incisée ou sculptée en bas-relief sur une plaque tournée vers le spectateur, suspendue entre les colonnes du dais, selon une typologie bien connue à Viterbe, Arezzo et Florence dans le dernier tiers du XIII $^{e}$ et le début du XIVe siècle ${ }^{8}$.

Un autre volet des études consacrées à Clément IV concerne le réalisme du gisant, à l'opposé de l'idéalisation des gisants français de la même époque. Monferini et Claussen ont exploré la piste dominicaine, Henriette S'Jacob celle de l'art antique ${ }^{9}$. Agostino Paravicini Bagliani a relié la représentation du gisant-cadavre aux théories sur la nature du pouvoir pontifical, 


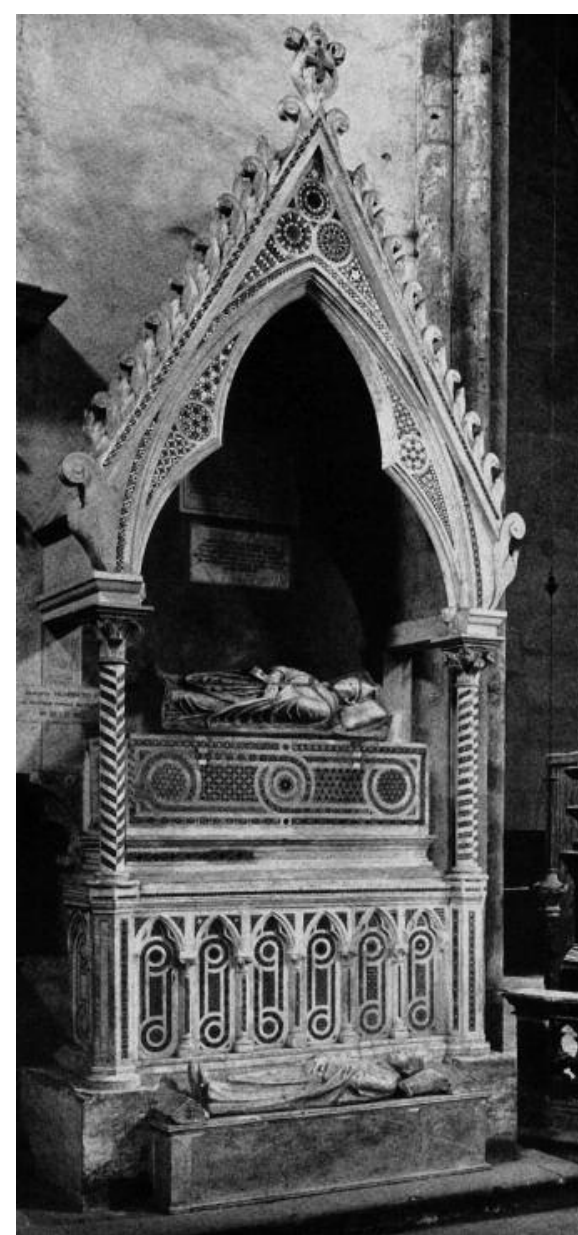

Cl. Gabinetto fotografico Nazionale, Rome. Fig. 1 - La tombe de Clément IV à San Francesco de Viterbe avant le bombardement de 1944. Le baldaquin est une restauration du XIXe siècle.

largement promues par les théologiens franciscains et dominicains et incarnées par une série de rites et de symboles visant à rappeler la caducité du pontife en tant qu'individu et la pérennité de l'Église ${ }^{10}$.

Le monument funéraire du pape français est donc loin d'être un champ d'étude vierge. Toutefois un aspect reste à approfondir : son emplacement originel dans l'organisation interne de l'église. Les publications nombreuses et conséquentes réalisées au cours des quarante dernières années se sont essentiellement focalisées sur des questions de style et de typologie. Cette situation est compréhensible puisque, jusqu'à une date récente, la réflexion développée dans le présent article aurait été difficile au vu de la maigreur des connaissances sur l'église du Duecento. Santa Maria in Gradi a été complètement reconstruite à partir de 1736 sous la direction de l'architecte Nicola Salvi et elle est aujourd'hui en ruines. Une première campagne d'études a été menée il y a une dizaine d'années, lors de la réhabilitation des bâtiments conventuels en vue de leur attribution à l'université de la Tuscia. Francesco Gandolfo avait alors émis quelques hypothèses sur le plan originel, mais il n'avait pas pu s'appuyer sur un relevé archéologique ${ }^{11}$. En 2007, Claudio Varagnoli est revenu sur le sujet, à partir de données archéologiques, archivistiques et de comparaisons avec d'autres églises dominicaines dans le Latium et en Ombrie ${ }^{12}$. Par conséquent, on ne dispose que depuis 2007 d'une étude solide sur l'organisation interne de l'édifice médiéval.

D'après la reconstitution de Varagnoli, l'église médiévale présentait un plan simple, s'inscrivant dans un rectangle, sans transept débordant. Le chevet comportait une grande abside rectangulaire flanquée de deux absidioles également rectangulaires (fig. 2). On sait d'après des sources antérieures à son premier déplacement que le monument de Clément IV était situé à l'origine contre le mur nord de l'abside centrale, à gauche de l'autel majeur ${ }^{13}$. Parmi les sources décrivant le couvent de Santa Maria in Gradi avant les travaux de Salvi, deux chroniques sont particulièrement précieuses. Toutes deux compilent des documents plus anciens aujourd'hui perdus ${ }^{14}$. La première est la Cronica compendiata de Giacinto Nobili. Écrite vers 1616 ou 1618, cette chronique est une version abrégée et en italien d'une chronique perdue que le même auteur avait rédigée en latin ${ }^{15}$. La seconde a été composée pour le chapitre général de Bologne en 1706 par le frère Francesco Maria Salmini ${ }^{16}$. À ces deux manuscrits, il faut ajouter une source repérée et publiée récemment par Massimo Miglio : une lettre anonyme de 1741 décrivant avec beaucoup de détails la destruction de l'église médiévale ${ }^{17}$. Ces trois textes sont clairs quant à l'emplacement de la tombe de Clément IV. Salmini écrit que le pape « repose du côté de l'Évangile par rapport à l'autel majeur, dans un célèbre sépulcre de pierre, orné de mosaïques " ${ }^{18}$ et la lettre anonyme de 1741 que les tombes de Pietro di Vico et du pape étaient « situées dans le pilier latéral des marches de l'autel majeur au fond du sanctuaire " ${ }^{19}$. Si la localisation de la tombe de Clément IV à gauche de l'autel majeur à l'époque moderne est donc certaine, la question n'est pas pour autant épuisée. En effet, cette position est étonnante, voire impossible, pour deux raisons qui incitent à approfondir le problème de l'emplacement de la tombe dans l'espace liturgique, à travers une enquête sur la localisation précise du chœur des frères et de l'autel majeur au XIII siècle.

Parmi les papes et cardinaux inhumés dans le sanctuaire d'une église dominicaine au XIII siècle, Clément IV serait un des rares étrangers à l'ordre. On observe en effet à cette époque une rigueur dans la répartition des sépultures chez les Prêcheurs : les seuls personnages inhumés dans le sanctuaire ou au milieu des stalles sont des membres importants de l'ordre ${ }^{20}$. Prenons l'exemple très représentatif des tombes cardinalices : à San Domenico d'Orvieto, le cardinal dominicain Annibaldo Annibaldi était inhumé devant l'autel majeur et les cardinaux Eudes de Châteauroux et Guillaume de Braye dans la nef ; à Notre-Dame de Confort à Lyon, Hugues de Saint-Cher était inhumé dans le sanctuaire mais Othon de Tonengo et Guillaume de Sabine de l'autre côté du jubé, etc. ${ }^{21}$. Ce n'est qu'à partir la fin du XIII siècle, puis surtout au cours du XIVe, que des étrangers à l'ordre furent ensevelis près du maître autel, dans l'ecclesia fratrum. À l'époque où la tombe de Clément IV fut réalisée, l'inhumation d'un étranger à l'ordre dans le sanctuaire était encore extrêmement rare chez les Prêcheurs. Le pape ne fut cependant pas le seul à bénéficier d'une sépulture privilégiée dans l'église de Santa Maria in Gradi. À la tombe du pontife, faisait pendant sur le mur sud du sanctuaire celle du préfet de Viterbe, Pietro di Vico, mort quelques jours après Clément IV, en décembre 1268. Pietro di Vico fut un grand bienfaiteur du couvent, comme en témoignent ses dispositions testamentaires ${ }^{22}$. Il demanda à être inhumé chez les Prêcheurs, sans réclamer cependant un emplacement précis dans l'église ${ }^{23}$. La 
tombe de Pietro di Vico constituerait alors un cas unique pour le XIII ${ }^{\mathrm{e}}$ siècle de sépulture laïque dans le sanctuaire d'une église dominicaine. En réalité, comme le montrera la démonstration ci-après, il n'en est rien.

L'exception faite pour Clément IV pourrait se justifier par l'importance du défunt pour l'ordre. Il semble en effet logique que les frères aient honoré par un emplacement privilégié une dépouille obtenue au terme d'une lutte de huit ans contre les chanoines. L'acharnement des Dominicains de Viterbe est compréhensible : le pape était très proche des Prêcheurs, puisqu'il passa les dernières années de sa vie dans leur couvent en suivant leur mode de vie. De plus, il mourut en odeur de sainteté : le chroniqueur Nobili décrit les miracles et les démonstrations de ferveur autour du corps avant l'inhumation ${ }^{24}$.

Dans les nombreux travaux qui lui sont consacrés, la tombe de Clément IV n'a jamais été considérée comme une tombe de saint, bien que les dévotions décrites par Nobili n'aient pas manqué d'être mentionnées. Le développement d'un culte autour des sépultures de bienheureux et de saints, parfois non canonisés officiellement, et ses incidences sur les formes artistiques ont été bien étudiés pour le milieu siennois du Trecento, à partir notamment du monument de Marguerite de Cortone ${ }^{25}$. La dévotion pour des saints contemporains a été encouragée à la fois par les ordres mendiants et par l'essor de l'identité communale ${ }^{26}$. Les tombeaux-reliquaires toscans du XIVe siècle ne se différencient pas, sur les plans typologique et iconographique, des tombes murales de prélats comme celle de Clément IV. Comme celles-ci, elles comportent un baldaquin, un gisant, une scène de présentation du défunt à la Vierge... Ce sont essentiellement les pratiques dévotionnelles autour du sépulcre qui permettent de le définir comme une tombe de saint, non des critères formels. C'est dans ce contexte dévotionnel et artistique extrêmement dynamique et complexe que se pose la question du statut du tombeau de Clément IV. Dans un article fondamental pour l'étude des tombes de saints en Italie,
«Gräber von Heiligen und Seligen », Jörg Garms évoque deux fois le problème, mais sans le trancher ${ }^{27}$. La question du statut de la tombe de Clément IV se pose pour Jörg Garms dans ses implications typologiques : il se demande si l'adoption inhabituelle de la solution du gisant n'avait pas pour but de manifester (ou de créer) la sainteté du défunt. Ce problème de la fama sanctitatis sera ici repris, non à partir de l'aspect

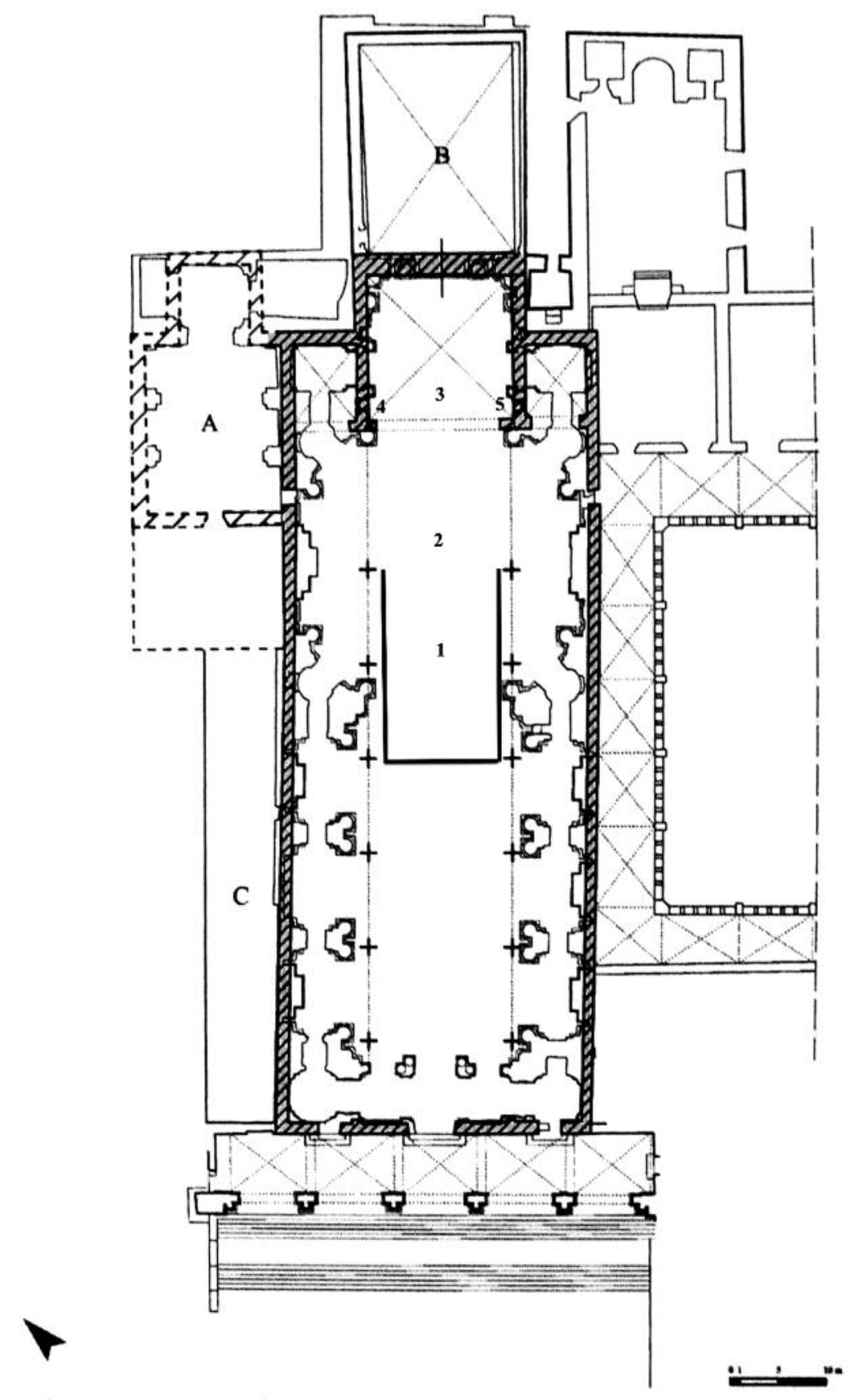

Fig. 2 - Viterbe, église Santa Maria in Gradi, plan avant la reconstruction du XVIII siècle, d'après Claudio Varagnoli (C. Varagnoli, "S. Maria in Gradi a Viterbo, dalla chiesa duecentesca al progetto di Nicola Salvi », Palladio, nº 40, juillet-décembre 2007. Le tracé des stalles et les numéros ont été ajoutés par l'auteur). 1 : Stalles des frères jusqu'en 1546 ; 2: Position de l'autel jusqu'en $1547 ; 3$ : Position de l'autel entre 1547 et $1741 ; 4$ : Tombe de Clément IV jusqu'en 1741 ; 5 : Tombe de Pietro di Vico jusqu'en 1741 ; B : Extension de l'abside construite à la fin du XVe siècle, emplacement des stalles à partir de 1546. 
typologique soulevé par Jörg Garms, mais à partir de la localisation du monument dans l'espace de Santa Maria in Gradi.

Il a été précédemment évoqué combien est surprenante la présence dans le sanctuaire de Santa Maria in Gradi de deux sépultures d'étrangers à l'ordre - dont un laïc qui plus est -, compte tenu de la politique funéraire des frères prêcheurs autour de 1268. Si l'emplacement des tombes de Pietro di Vico et de Clément IV ne peut être mis en doute, ne faut-il pas alors reporter le problème sur la position de l'autel ? La dévotion populaire autour de la sépulture pontificale est un autre élément qui pousse à reconsidérer l'organisation de l'église au Moyen Âge. La description de miracles autour de la dépouille du pontife avant son inhumation ne constitue pas en soi la preuve d'un réel et durable culte puisque ce thème est un topos des biographies pontificales à partir d'Honorius III $(\dagger 1227)^{28}$. En revanche, Giacinto Nobili donne plusieurs indices démontrant qu'une dévotion persista plusieurs années autour du tombeau de Clément IV : il était entouré d'une grille, il portait des traces de vandalisme comme si l'on avait voulu en soustraire quelque reliques et de vieux exvoto y étaient accrochés ${ }^{29}$. Ces éléments ne laissent donc aucun doute sur le fait que les fidèles pouvaient s'approcher du monument. Par conséquent, ce dernier ne pouvait pas se trouver dans l'ecclesia fratrum qui n'était pas accessible aux fidèles. Sur ce point, les actes capitulaires et le commentaire de la règle par le général de l'ordre Humbert de Romans sont très clairs : ils imposent la présence d'un jubé (intermedium) séparant les frères des fidèles et rendant invisibles depuis la nef les déplacements des religieux entre le cloître et le chœur liturgique. Le franchissement de l'intermedium n'était consenti qu'à de rares occasions, à certains laïcs du sexe masculin revêtant une certaine dignité, sous contrôle du chantre ${ }^{30}$. Les femmes, même les nobles bienfaitrices, ne pouvaient en aucun cas accéder à la salle du chapitre ou à la zone du chœur liturgique et du sanctuaire.

Une telle rigueur sur l'interdiction de l'accès à l'ecclesia fratrum pour les laïcs laisse perplexe quant à la possibilité d'une dévotion autour de la tombe de Clément IV. Le problème peut cependant être résolu si l'on considère de plus près l'organisation interne de l'église au Duecento. Toutes les sources signalant la présence des tombes de Clément IV et de Pietro di Vico de part et d'autre de l'autel majeur datent des XVII et XVIII ${ }^{\mathrm{e}}$ siècles. Or, à cette époque, l'autel majeur n'était plus à son emplacement originel mais plus à l'est par rapport à celui-ci. La chronique de Giacinto Nobili dit en effet précisément que, au cours du XVI ${ }^{\mathrm{e}}$ siècle, l'autel fut décalé vers l'abside et le chœur des frères déplacé de l'ouest de l'autel à l'est, dans une extension de l'abside construite dans la deuxième moitié $\mathrm{du} X \mathrm{XV}^{\mathrm{e}}$ siècle. $\mathrm{La}$ Cronica compendiata fixe en 1505 le début des travaux destinés à enlever les anciennes stalles qui se trouvaient " entre les trois colonnes plus proches de l'autel majeur ". La construction des nouvelles stalles fut commencée après les travaux d'allongement de l'abside. Le chantier fut ralenti par les exactions des soldats de Charles Quint en 1527 et finalement achevé en $1546{ }^{31}$. Lannée suivante, l'autel majeur fut déplacé vers les nouvelles stalles, donc vers l'est; à cette occasion, furent retrouvées les reliques placées dans l'autel trois siècles plus tôt lors de la première consécration par Alexandre IV ${ }^{32}$. On peut donc reconstituer la disposition suivante pour la période XIII$\mathrm{XVI}^{\mathrm{e}}$ siècle. Les stalles des frères se trouvaient dans les deux dernières travées orientales du vaisseau central de la nef (" entre les trois colonnes les plus proches de l'autel majeur " écrit Nobili). L'autel majeur était logiquement au centre de la travée en avant de l'abside puisque, après avoir été déplacé vers l'est, il se retrouva à l'entrée de l'abside, entre les monuments funéraires de Clément IV et Pietro di Vico (fig. 2).

La transformation du chœur liturgique de Santa Maria in Gradi s'inscrit dans un mouvement général. Bien qu’il n'existe pas à ce jour de synthèse sur le déplacement à l'époque moderne des chœurs monastiques, canoniaux et conventuels derrière l'autel majeur, on dispose de deux importantes contributions sur le sujet, publiées par Donal Cooper et Sible de Blaauw ${ }^{33}$. Si la modification de la position des stalles par rapport à l'autel fut effectuée systématique- ment après le Concile de Trente, le mouvement avait été amorcé par plusieurs églises dès le XVe siècle, voire dès le XIII ${ }^{\mathrm{e}}$ siècle pour un groupe d'églises franciscaines d'Ombrie ${ }^{34}$. En Italie centrale, il semble que Santa Maria in Gradi représente un cas précoce de déplacement des stalles - cette impression mériterait toutefois d'être étayée par une étude exhaustive des modifications portées à l'aménagement des églises autour du Concile de Trente. À Florence par exemple, c'est seulement en 1565 que furent lancés à Santa Maria Novella les travaux de réaménagement de l'édifice ; le projet confié par Cosme de Médicis à Vasari comprenait entre autres la destruction du jubé et le déplacement de l'autel vers l'ouest pour installer les stalles, initialement situées dans la nef, derrière l'autel, dans l'abside ${ }^{35}$. Santa Maria Novella fait figure de pionnière dans la ville des Médicis : les autres églises conventuelles, monastiques ou canoniales lui emboîtèrent ensuite le pas.

La disposition de l'église florentine était à l'origine différente de celle de Santa Maria in Gradi : l'autel majeur de Santa Maria Novella se trouvait dans l'abside avant d'être déplacé vers l'ouest pour laisser l'espace aux stalles, alors que celui de Santa Maria in Gradi fut rapproché du nouveau chœur des frères, donc décalé vers l'est. Il est notable qu'à l'origine, l'autel majeur de l'église dominicaine de Viterbe se soit trouvé dans la travée la plus orientale de la nef, laissant libre tout l'espace de l'abside. Cette disposition est surprenante : dans les églises mendiantes dotées d'une abside, l'autel ne se trouvait dans la nef ou à la croisée du transept (pour les églises qui en étaient pourvues) que dans le cas particulier, étudié par Donal Cooper, des églises franciscaines dotées d'un rétro-chœur. À Viterbe, les sources sont assez claires quant à la position originelle des stalles pour exclure un rétrochœur. Il est par conséquent tentant de voir dans le décalage de l'autel majeur et des stalles vers la nef la preuve d'une attribution délibérée de l'abside à une fonction funéraire.

On peut en tirer les conclusions suivantes. Les tombes de Clément IV et de Pietro di Vico ne se sont trouvées de part et 
d'autre de l'autel majeur qu'à partir de 1547, puisque l'autel était à l'origine dans la dernière travée de la nef. Le problème qui s'est posé au départ est alors résolu : la partie fermée aux fidèles, l'ecclesia fratrum, était circonscrite aux trois travées orientales du vaisseau central de la nef. Par les bascôtés, les fidèles pouvaient circuler entre la nef et la chapelle axiale. Plus précisément, on peut supposer que la circulation des fidèles se faisait par le bas-côté nord, puisque la porte vers le cloître s'ouvrait dans le mur sud du transept et que les mouvements des frères entre le cloître et le chœur liturgique devaient rester invisibles aux fidèles ${ }^{36}$. Un accès des laïcs au bas-côté nord semble donc la seule solution envisageable pour expliquer la possibilité d'une dévotion autour du tombeau de Clément IV. Une telle disposition n'était cependant pas en pleine conformité avec les normes de l'ordre puisque, selon le chapitre général de 1249, il était interdit de laisser les femmes circuler dans les bascôtés de part et d'autre des stalles ${ }^{37}$. Une entorse à cette règle semble toutefois mineure comparée à celle qu'aurait représenté un accès des laïcs au sanctuaire. Le couvent de Viterbe a probablement pris une certaine liberté par rapport aux prescriptions, liberté justifiée par la présence du tombeau d'un saint ${ }^{38}$. Un autre doute demeure cependant à propos de l'organisation interne de Santa Maria in Gradi : existait-il un jubé ? Aucun document n'en fait mention. Si les textes législatifs dominicains imposent un intermedium pour séparer les frères des fidèles, celui-ci a pris des formes diverses : paroi en pierre ou en bois sur deux niveaux, simple balustrade faisant le tour du chœur liturgique... Les stalles des frères jouaient également parfois le rôle d'intermedium, ce qui pourrait avoir été le cas à Viterbe ${ }^{39}$.

Ces éclaircissements sur la position originelle du monument funéraire de Clément IV dans l'espace de Santa Maria in Gradi apportent donc une double contribution à l'étude de l'aménagement des églises dominicaines. Premièrement, ils révèlent une organisation interne de Santa Maria in Gradi adaptée à la promotion du culte d'un saint contemporain. Deuxièmement, il semble que l'église dominicaine de Viterbe ait fait l'objet d'un réaménagement de la zone orientale précoce, dès la fin du $X V^{e}$ siècle - je laisse ce deuxième point aux historiens de l'architecture et de la liturgie modernes. La tombe de Clément IV peut ainsi légitimement être incluse dans l'histoire des tombeaux de saints, sans les réserves exprimées par J. Garms, même si rien sur le plan formel ne la distingue comme telle. En réalité, comme l'ont montré notamment les études de Joanna Cannon, le développement d'une nouvelle sainteté au XIII ${ }^{\mathrm{e}}$ siècle, en milieu mendiant, conduisit à la naissance d'un type original de tombeau de saint qui ne se distinguait pas tant par sa typologie que par sa position dans l'édifice et par les rites dont il était le centre (processions, messes, approche par les fidèles malades) ${ }^{40}$.

La tombe de Clément IV peut être mise en regard avec deux autres monuments de saints dominicains dont la

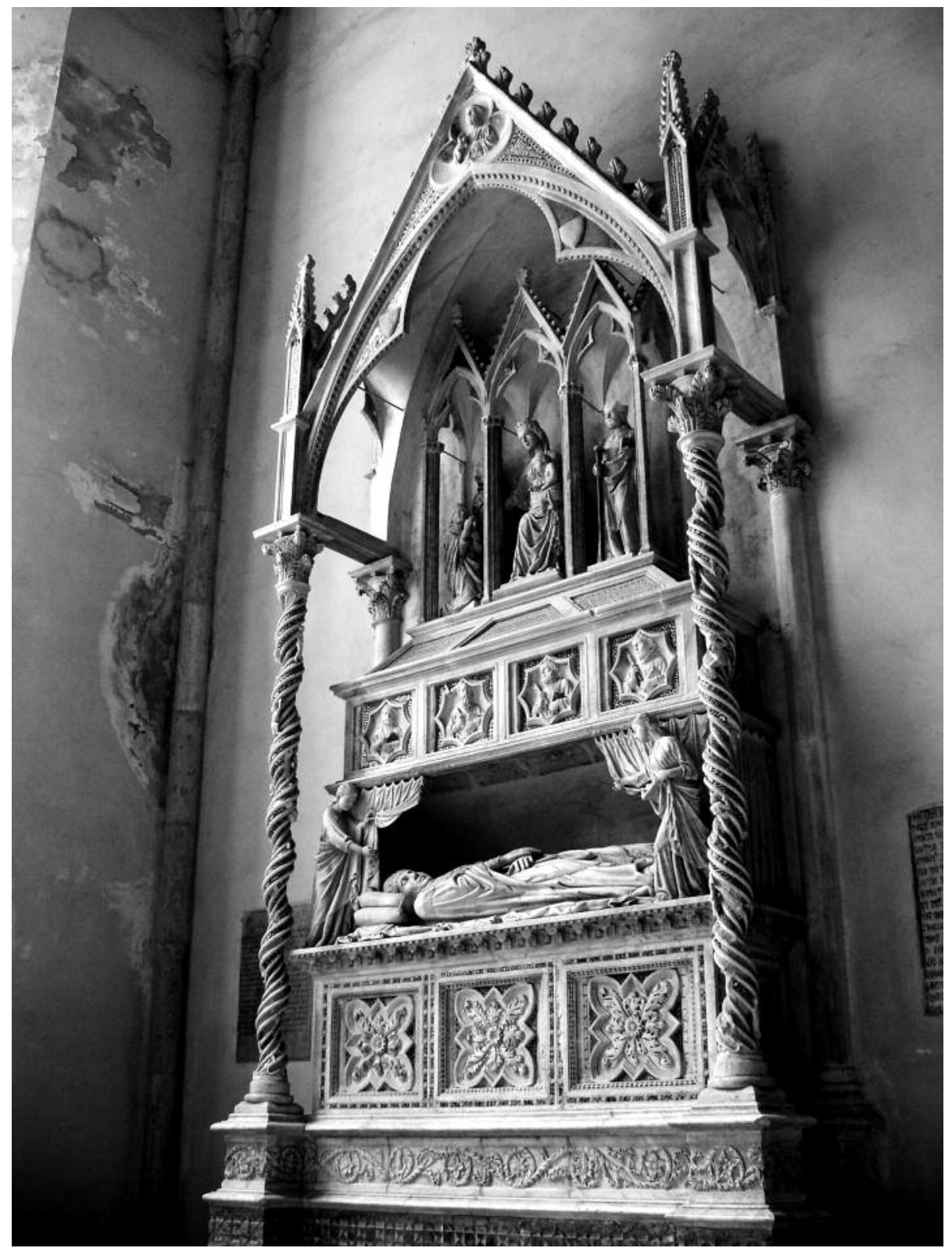

Cl. H. Morvan.

Fig. 3 - Tombe de Benoît XI († 1304) à San Domenico de Pérouse. 
position fut également soigneusement choisie afin de favoriser la dévotion populaire. La tombe de saint Dominique à Bologne constitue un exemple paradigmatique de l'évolution de la politique des Prêcheurs pour la promotion des saints de l'ordre. Les frères n'ont cherché à rendre accessible aux fidèles la tombe de leur fondateur qu'à partir de 1233, après une première période de refus de la dévotion populaire - les frères de Bologne allèrent même jusqu'à détruire les ex-voto ${ }^{41}$. En 1233, la précieuse dépouille fut déplacée depuis le chour dans le bas-côté sud. La lettre encyclique de Jourdain de Saxe écrite à cette occasion est explicite quant aux buts de la translation : mettre le corps à l'abri (la zone absidale était alors en travaux) et lui donner son utilité, c'est-à-dire le rendre accessible aux fidèles venus réclamer les grâces du saint. Le général de l'ordre présente même cette mise en valeur du Maria in Gradi di Viterbo. Archivio della Provincia Romana (Santa Maria sopra Minerva), F.IV.11. G. Nobili, Chronique abrégée de Santa Maria in Gradi de Viterbe

p. 11 : Consacrò anche questo Pontefice l'Altar maggiore et pose in quello molte Reliquie de Santi, le quali nel transportar detto Altare un poco più sù, verso il novo Coro, furon trovate l'anno 1547 (...). (Della sacra della Chiesa. Capitolo quarto)

Ce pape [Alexandre IV] consacra aussi l'autel majeur et il y posa de nombreuses reliques de saints, lesquelles furent trouvées l'an 1547, lors du déplacement dudit autel un peu plus haut, vers le nouveau chœur. (De la consécration de l'église. Chapitre IV)

p. 22 : Il Coro fu incominciato da Frati l'anno 1505 per levare il vecchio, che stava tra le tre colonne più vicine all'Altar maggiore. Ma nell'anno 1527 essendo condotti li travi, tavole et altro legname necessario per l'Armature, etc. di detta fabrica, furon tutti abrugiati dalli soldati, che andorno al sacco di Roma, onde convenendo di novo provedere si trattene l'opera sino al'anno 1546, in cui fu totalmente compito di edificare. (Di alcune fabriche della Chiesa. Capitolo decimo)

Le chœur fut commencé par les frères l'an 1505 pour enlever l'ancien qui était entre les trois corps comme l'accomplissement de la volonté divine à laquelle les frères étaient jusque là restés sourds :

"Iacebat nempe thesaurus absconditus, carens utilitate, ut subtrabebantur beneficia desuper a virtutum largitore ; iusticie enim equitas exigebat hiis graciam subrahi, qui graciam dei et gloriam occultare nitebantur $"{ }^{42}$.

En 1267, le monument commandé trois ans plus tôt à Nicola Pisano fut installé juste à l'ouest du jubé, de manière à être visible des fidèles mais également proche de la communauté des frères.

La sépulture du pape dominicain Benoît XI († 1304) à San Domenico de Pérouse constitue un autre exemple significatif de solution adoptée par les Dominicains pour encourager la dévotion à une personnalité issue de l'ordre (fig. 3) ${ }^{43}$. Le pape Benoît XI, béatifié seulement au XVIII siècle, fut considéré comme saint dès sa mort ${ }^{44}$. Il fut enseveli, selon son souhait,

\section{ANNEXE}

\section{LA CHRONIQUE ABRÉGÉE DE GIACINTO NobILI}

colonnes plus proches de l'autel majeur. Mais, au cours de l'an 1527, alors qu'avaient été apportés les poutres, les planches et autre bois nécessaire pour l'armature, etc. de cette construction, tout ceci fut brûlé par les soldats qui allaient au sac de Rome. C'est pourquoi, une fois qu'on eut convenu de le reprendre, le chantier se prolongea jusqu'en l'an 1546, au cours duquel on acheva totalement la construction. (De quelquesuns des travaux de l'église. Chapitre X)

p. 26 Il corpo suo essendo posto secondo il solito in Chiesa, Dio per li meriti del santo Pontefice operò molti, et rari miracoli ; onde concorendo i Popoli à visitarlo, toccarlo, et bagiarlo per ottener col suo mezzo gratie, et favori da sua Divina Maestà. (Delli homini segnalati sepolti nella Chiesa. Capitolo undecimo)

Son corps [à Clément IV] étant placé selon l'habitude dans l'église, Dieu par les mérites du saint pontife opéra de nombreux et rares miracles. C'est pourquoi le peuple accourut pour le visiter, le toucher, et l'embrasser pour obtenir par son intermédiaire grâces et faveurs de sa divine majesté. (Des hommes illustres inhumés dans l'église. Chapitre XI)

p. 27 : Finalmente doppo longa lite di sette anni in circa fu con sommo honore, si come meritava un'huomo tanto degno, et grato à Dio traportato in Gradi, ove si conserva à guisa d'una delle maggiore gioie, et tesori, che habbia la Religione sous une tombe plate devant l'autel. C'est probablement aux frères pérugins qu'il faut attribuer l'initiative de la commande d'un cénotaphe monumental, sur le modèle des tombes sculptées par Arnolfo di Cambio (notamment celle du cardinal Guillaume de Braye à Orvieto), au milieu d'un mur latéral de la nef. Le pape avait donc sa sépulture réelle dans l'ecclesia fratrum et un cénotaphe destiné à la dévotion des fidèles dans l'ecclesia laicorum. Le gisant du cénotaphe manifestait pour le fidèle la présence du corps qui se trouvait en réalité de l'autre côté du jubé. L'absence de contact direct avec les reliques, ou au moins avec leur contenant, n'empêcha pas la ferveur des fidèles.

À Viterbe en revanche, les frères conçurent un aménagement de l'espace original permettant aux fidèles d'accéder à la sépulture réelle de Clément IV, non à un simple cénotaphe.
Dominicana in un sepolcro di marmo fino, lavorato tutto di mosaico con cancellata di ferro intorno, sopra di cui in pietra con versi all'antica, si raconta la sua vita. Si vede, che più volte è stato tentato di rompere, o aprire in qualche luogho la cassa, ove sta sepolto, per vederlo, o per cavarne qualche Reliquia, ma anche non si è penetrato al suo luogho. Molti voti, imagini, et tavolette in segno delle gratie ricevute per meriti del Santo Pontefice erano appesi al sepolcro, quali per antichità son caduti. (Delli homini segnalati sepolti nella Chiesa. Capitolo undecimo)

Enfin, après une longue dispute de sept ans environ, avec grand honneur, comme le méritait un homme si digne et aimé de Dieu, il [Clément IV] fut transporté à Gradi, où il est conservé comme l'une des plus grandes joies et trésors que possède l'ordre dominicain, dans un sépulcre de marbre fin, tout travaillé de mosaïques avec une grille de fer autour, audessus duquel en pierre avec des vers à l'antique on raconte sa vie. On voit que, plusieurs fois, on a tenté de casser ou d'ouvrir en quelque endroit le sarcophage où il est enseveli pour le voir ou pour en tirer quelque relique; mais on n’a pas réussi à y pénétrer. De nombreux vœux, images et tablettes, en signe des grâces reçues par les mérites du Saint Pontife, étaient accrochés au sépulcre, et sont tombés avec le temps. (Des hommes illustres inhumés dans l'église. Chapitre XI) 


\section{NOTES}

* Haude Morvan achève un doctorat en histoire de l'art médiéval en cotutelle Paris IV-Sorbonne - Roma La Sapienza. Elle a parallèlement été admise en cycle de Perfezionamento ) la Sculo Normale Superiore de Pise.

1. A.M. D’Achille, «Sulla datazione del monumento funebre di Clemente IV a Viterbo : un riesame delle fonti ", Arte Medievale, s.II n 3, 1989, p. 85$91 ;$ id., «Il monumento funebre di Clemente IV in San Francesco a Viterbo ", dans J. Garms et A. M. Romanini (dir.), Skulptur und Grabmal des Spätmittelalters in Rom und Italien, Vienne, 1990 p. 129-142 ; id., "Le sepolture medievali ", dans M. Miglio (dir.), Santa Maria in Gradi, Viterbe, 1996, p. 129-159 ; id., Da Pietro d'Oderisio ad Arnolfo di Cambio : studi sulla scultura a Roma nel Duecento, Rome, 2000, p. 98.

2. P.C. Claussen, Magistri doctissimi romani. Die römischen Marmorkünstler des Mittelalters, Stuttgart, 1987, p. 196-199 ; id., "Pietro di Oderisio und die Neuformulierung des italienischen Grabmals zwischen Opus romanum und Opus francigenum ", dans Skulptur und Grabmal des Spätmittelalters in Rom und Italien, J. Garms et A. M. Romanini (dir.), Vienne, 1990, p. 173-207.

3. J. Gardner, «Arnolfo di Cambio and Roman Tomb Design ", The Burlington Magazine, $\mathrm{n}^{\circ}$ 115, 1973 p. 420-439, p. 424-427; id., The tomb and the tiara, Oxford, 1992 , p. 36 et p. 48.

4. Voir notamment I. Herklotz, « Sepulcra » e « monumenta " del Medioevo : studi sull'arte sepolcrale in Italia, Napoli, 2001 (1e édition à Rome en 1985), p. 205 293 (I primi sepolcri con la figura del defunto).

5. Biblioteca comunale degli Ardenti de Viterbe, Ms II.C.IV.43, p. 57. Le dessin a été publié à plusieurs reprises : A. M. D'Achille, "Le sepolture medievali », op. cit. note 1, p. 155 ; I. Herklotz, op. cit. note 4, p. 247.

6. I. Herklotz (op. cit. note 4, p. 246-248) considère le dessin du tombeau de Paolo di Pafo comme un importante argomento che potrebbe privare la tomba di Clemente anche del suo ruolo di primo monumento con la figura giacente. Anna D'Achille considère en revanche qu'étant donné l'imprécision du dessin et l'incertitude sur les dates de réalisation des monuments, la prudence s'impose (A. M. D'Achille, « Le sepolture medievali ", op. cit. note 1, p. 77).

7. S. Pacella, « Le tombe di Ruggero Duraldi e Paolo di Pafo in Santa Maria in Gradi. Una nuova fonte ", Rivista storica del Lazio, année VII, $\mathrm{n}^{\circ} 12,2000$, p. 3-9.

8. Sur cette typologie de tombe, voir F. Schwartz, «Die Memoria bei den Fratres : das Grabmal des Fra Aldobrandino Cavalcanti und ein dominikanischer Typus für Bischofsgrabmäler ", dans W. Maier, W. Schmid et M.V. Schwarz (dir.), Grabmäler : Tendenzen der Forschung an Beispielen aus Mittelalter und früher Neuzeit, Actes du congrès de Trier (24-26 septembre 1997), Berlin, 2000, p. 201-229.

9. A. Monferini, «Pietro di Oderisio e il rinnovamento tomistico ", dans Momenti del marmo, scritti per i duecenti anni dell'Accademia di Carrara, Rome, 1969, p. 39-63 ; P.C. Claussen, " Pietro di Oderisio... " cit. note 2 ; H. S'Jacob, Idealism and realism, a study of sepulcral symbolism, Leiden, 1954, p. 28.

10. A. Paravicini Bagliani, Il corpo del papa, Torino, 1994, p. 201 et passim; idem, « Rileggendo i testi sulla 'nudità del papa' ", dans Il potere del papa. Corporeità, autorappresentazione, simboli, Florence, 2009, p. 2144, p. 32.

11. F. Gandolfo, "La vincenda edilizia ", dans M. Miglio (dir.), Santa Maria in Gradi, Viterbo, 1996, p. 41-93.

12. C. Varagnoli, «S. Maria in Gradi a Viterbo, dalla chiesa duecentesca al progetto di Nicola Salvi ", Palladio, $n^{\circ} 40$, juillet-décembre 2007, p. 5-26.

13. L'hypothèse de Cristofori d'un emplacement sur la contre-façade est sans fondement (F. Cristofori, $L e$ tombe dei papi in Viterbo, e le chiese di S. Maria in Gradi, di S. Francesco e di S. Lorenzo. Memorie e documenti sulla storia medioevale Viterbese, Siena, 1887, p. 32).

14. Sur les chroniques conservées et perdues du couvent de Santa Maria in Gradi, voir E. Panella, «Cronaca antica di Santa Maria in Gradi di Viterbo : perduta o mai esistita ? ", Archivum Fratrum Praedicatorum, $\mathrm{n}^{\circ} 65,1995$, p. 185-233.

15. G. Nobili, Cronica compendiata di Santa Maria in Gradi di Viterbo. Archivio della Provincia Romana (Santa Maria sopra Minerva), F.IV.11.

16. F.M. Salmini, Cronologia Gradensis seu conventus Sanctae Mariae ad Gradus de Viterbio. Archivio Generale dell'Ordine dei Predicatori, XIV.C.I à partir $\mathrm{du} \mathrm{f}^{\mathrm{7}} 71$. Les folios du volume correspondant à la chronique de Salmini portent trois systèmes de numérotation : $1^{\circ}$ les folios de la chronique, $2^{\circ}$ les pages de tout le volume XIV.C, $3^{\circ}$ les pages propres à la Cronologia gradensis. Les références à la Cronologia dans cet article se rapportent à cette troisième numérotation, correspondant aux chiffres en haut à gauche de chaque page.

17. Archivio Segreto Vaticano, Fondo Domenicani, II, 42, cc.79-81. Publié dans M. Miglio, "Una ricognizione della tomba di Clemente IV ", Rivista storica del Lazio, n 5, 1996, p. 41-44.

18. Cronologia Gradensis, op. cit. note 16, p. 119 : jacet ad latus Evangelii Maioris Ares in lapideo ac celebri sepulcro, opere musivo exornatus.

19. Fu espediente trasferire li depositi di Pietro di Vico, e suoi, e di Clemente papa IV, quali erano situati nel pilastro laterale delli scalini dell'altare maggiore in fondo al presbiterio (voir note 17).

20. Font exception à cette règle quelques évêques français qui reçurent une tombe dans le sanctuaire. Ces derniers n'étaient pas dominicains mais furent à l'origine de la fondation du couvent dans leur diocèse, ce qui explique l'inhumation privilégiée. On peut ainsi mentionner : Hugues de la Ferté (†1236) à Chartres, inhumé sous un monument avec gisant,
Bernard de Montaigu ( $† 1248)$ au Puy, sous un enfeu avec gisant, Michel de Villoiseau ( $\dagger$ 1260) à Angers sous une tombe émaillée au milieu du chœur, Jean d'Upsal (†1291) en bas des marches du grand autel de Provins. À la suite de Hugues de la Ferté, la plupart des évêques chartrains se firent enterrer chez les Dominicains, l'inhumation dans la cathédrale étant interdite. Voir G. Rohault de Fleury, Gallia dominicana. Les couvents de saint Dominique au Moyen Âge, Paris, 1903 (ouvrage sans pagination) ; J. Adhémar et G. Dordor, "Les tombeaux de la collection Gaignières ", Gazette des Beaux-Arts, no 84 (1974), p. 1-92, nos 38, 55 ; M. Négri, « Les Mendiants et la mort en milieu chartrain à l'époque médiévale ", Bulletin de la société archéologique d'Eure-et-Loire, $n^{\circ} 91$ (2007), p. 1-38 et $i d$., "Les sépultures médiévales des deux couvents mendiants chartrains ", Bulletin de la société archéologique d'Eure-et-Loir, $\mathrm{n}^{\circ} 92$ (2007), p. 11-54.

21. Cette question, ici rapidement évoquée, est l'un des principaux points de ma thèse de doctorat qui sera soutenue courant 2013 (cotutelle Paris IV-Sorbonne et Roma I-Sapienza). Sur le cas lyonnais, voir H. Morvan, "La morte nella propaganda domenicana : le tombe cardinalizie ed ipredicatori lionesi nel Duecento ", Memorie domenicana, $\mathrm{n}^{\circ}$ 42, 2012, p. $577-592$.

22. On conserve un codicille au testament de Pietro di Vico, publié dans F. Bussi, Istoria della città di Viterbo, Rome, 1742, p. 410-411.

23. Id., In Ecclesia praedicta suam elegit humiliter sepulturam

24. Cronica compendiata, op. cit. note 15, p. 26 (voir le passage et sa traduction en annexe).

25. G. Bardotti Biasion, «Gano di Fazio e la tombaaltare di Santa Margherita da Cortona ", Prospettiva, $\mathrm{n}^{\circ} 37,1984$, p. 2-19 ; J. Garms, "Gräber von Heilige und Seligen » dans J. Garms et A.M. Romanini (dir.), Skulptur und Grabmal des Spätmittelalters in Rom und Italien, Wien, 1990, p. 83-105 ; J. Cannon, « Popular saints and private chantries. The sienese tomb-altar of Margherita of Cortona and questions of liturgical use ", dans N. Bock, S. de Blaauw (dir.), Kunst und Liturgie im Mittelalter, Akten des internationalen Kongresses der Bibliotheca Hertziana und des Nederlands Instituut te Rome (Rome, 28-30 septembre 1997), Münche, 2000, p. 149-162.

26. A. Vauchez, « La commune de Sienne, les Ordres Mendiants et le culte des Saints. Histoire et enseignement d'une crise ", dans Mélanges de l'École Française de Rome, Moyen Âge, $\mathrm{n}^{\circ}$ 8, 1977, p. 757 767 ; id., La sainteté en Occident aux derniers siècles du Moyen Age (1198-1431), Rome, 1981 ; M. Goodich, Vita perfecta: The ideal of sainthood in the thirteenth century, Stuttgart, 1982, p. 43-44, p. 149 ; M. Tomasi, «Il modello antoniano : tombe di santi su colonne o su cariatidi ", Il Santo, $\mathrm{n}^{\circ} 48,2008$, p. 179-200, p. 185-187.

27. J. Garms, "Gräber von Heilige... », op. cit. note 25, p. 96 : Interessanter erscheint die Frage, ob nicht bei Auftrag und Form des Grabes Klemen'IV., ehemals in der Dominikanerkirche S. Maria in Gradi vor Viterbo, 
Überlegungen in Richtung auf einen eventuellen Kult mitgespielt haben könnten - wäre dies der Fall, würde das Problem, ob es sich hiebei wirklich um das erste italienische Monumentalgrab mit Liegefigur handelt, eine weitere Dimension erhalten. Anhaltspunkte dazu gibt der Bericht über den Streit um den Leichnam des toten Papstes zwischen den Dominikanern und den Domkanonikern mit der Erwähnung eines Volksauflaufes wegen der Heiligkeit und der Wunder des Verstorbenen einerseits, die Rolle des damals in Viterbo weilenden Ordensgenerals Giovanni da Vercelli andererseits, der schon die treibende Kraft hinter der Ausführung des Dominikus-Grabes in Bologna gewesen war. et p. 100 : Der Fall Klemens'IV. wurde schon erwähnt - problematisch auch insofern, als die Intention, hier das Mal eines künftigen Heiligen zu errichten, möglich erscheint.

28. A. Paravicini Bagliani, Il corpo del papa, Torino, 1994, p. 199-203.

29. Cronica compendiata, op. cit. note 15, p. 26 (voir le passage et sa traduction en annexe).

30. «Quand des étrangers entrent dans le choeur, ou se mettent devant la porte, il revient à lui [le chantre], selon leur degré de dignité, de les amener au lieu approprié. Si ce sont des personnes humbles, il doit les éloigner poliment des sièges les plus dignes, ou carrément les éloigner du chœur, s'il peut le faire sans scandale. C'est lui également qui est chargé d'empêcher les étrangers d'entrer trop facilement dans le choeur, et surtout les laïcs et les enfants. » Traduction de l'auteur d'après l'édition du texte latin : Humbert de Romans, De vita regulari, Berthier (éd.) vol. II, 1889, p. 242. Chapitre général de Trêve en 1249: «Que les jubés qui sont dans nos églises entre les laïcs et les frères soient disposés partout par les prieurs de manière à ce que les frères lorsqu'ils entrent et sortent du chœur ne puissent pas être vus par les fidèles, ou voir ceux-ci. Ils pourront cependant y aménager des fenêtres pour qu'elles puissent être ouvertes au moment de l'Elévation du corps du Christ. ". Traduction de l'auteur d'après l'édition du texte latin : B.M. Reichert (éd.), Acta Capitulorum generalium ordinis praedicatorum, vol. I (ab anno 1220 usque ad annum 1303), Romae, 1898 (Monumenta Ordinis Praedicatorum Historica t. III), p. 47. Voir aussi C.G. Gilardi, "Ecclesia laicorum e ecclesia fratrum. Luoghi e oggetti per il culto e la predicazione secondo l'Ecclesiasticum officium dei frati predicatori ", dans L.E. Boyle et P.-M. Gy (dir.), Aux origines de la liturgie dominicaine : le manuscrit Santa Sabina XIV L1, Rome, 2004, p. 379-443, p. 415 note 123 et p. 434 note 198 .

31. Cronica compendiata, op. cit. note 15, p. 22 (voir le passage et sa traduction en annexe).
32. Cronica compendiata, op. cit. note 15, p. 11 (voir le passage et sa traduction en annexe).

33. D. Cooper, « Franciscan choir encolsures and the function of double-sided altarpieces in pre-Tridentine Umbria ", Journal of the Warburg and Courtauld Institutes, $\mathrm{n}^{\circ}$ 64, 2001, p. 1-54 ; S. De Blaauw, «Innovazioni nello spazio di culto fra basso Medioevo e Cinquecento : la perdita dell'orientamento liturgico e la liberazione della navata ", dans J. Stabenow (dir.), Lo spazio e il culto. Relazioni tra edificio ecclesiale e uso liturgico dal XV al XVI secolo, journées d'étude au Kunsthistorisches Institut de Florence (27-28 mars 2003), Venezia, 2006, p. 25-51, p. 38-43. Voir aussi C.G. Gilardi, op. cit. note 30, p. 441-442.

34. L'article de Donal Cooper (voir note 33) traite justement de ce cas particulier du rétro-chœur, attesté avec certitude ou par des indices très probants pour un groupe d'églises franciscaines d'Ombrie. L'auteur développe les liens de cette disposition avec la commande de retables peints sur les deux faces. Selon lui, cette organisation originale est à comprendre à la lumière du modèle prestigieux de la basilique d'Assise.

35. R. Lunardi, "Ristrutturazione vasariana di S. Maria Novella ", Memorie domenicane, n ${ }^{\circ}$ 19, 1988, p. 403-419, en particulier, sur le chœur, p. 406-410. Voir aussi le commentaire d'un observateur contemporain, A. Lapini, Diario fiorentino dal 252 al 1596, O. Corazzini (éd), Firenze, 1900, p. 152 : E li sopradetti frati domenicani furono i primi a levare detto coro e ponte che era nel mezzo della chiesa, e tornarono dietro l'altare maggiore e vi fecero fare allora il bel coro che vi si vede, che fu cosa che piacque molto a l'universale, l'aver levato detti cori.

36. Voir note 30. Cette disposition demeure valide à l'époque moderne puisque, lors du réaménagement du chœur à Santa Maria Novella en 1565-1566, il fut prévu que les mouvements des frères entre le dortoir et le chœur restent invisibles aux fidèles. Lunardi (op. cit. note 35, p. 408) reproduit une note envoyée au duc Cosme de Médicis résumant les travaux à faire : Fare il coro doppio per i frati, assettarlo non levando le spalliere, né alterando la cappella, né dipinture, come sarà giudicato da l'ingegneri, con far l'entrata dietro alle cappelle che si possa di dormitorio venire in choro senza che i frati siano visti.

37. In alis quae sunt in ecclesiis juxta chorum fratrum a dextris et a sinistris mulieres ingredi non permittantur (Acta Capitulorum generalium... op. cit. note 30, p. 47).

38. C.G. Gilardi a démontré brillamment comment l'esprit de la législation des Prêcheurs repose dès l'origine sur une certaine flexibilité, permettant une adap- tation rapide des couvents au contexte (Gilardi, op. cit. note 30 ).

39. La question des jubés dans les églises mendiantes fait encore l'objet de nombreux débats. Voir : M. Hall, "The tramezzo in Santa Croce, Florence, Reconstructed ", Art Bulletin, nos 56-3 (1974), p. 325341 ; M. Merotto Ghedini, «Il tramezzo nella chiesa dei santi Giovanni e Paolo a Venezia ", dans T. Franco (dir.), De Lapidibus sententiae, scritti di storia dell'arte per Giovanni Lorenzoni, Padova, 2002, p. 257-262 ; J. Barclay-Lloyd, « Medieval domenican architecture at Santa Sabine in Rome c.1219-1320 ", Papers of the British School at Rome, n 72, 2004, p. 231-292, en particulier p. 251-259 ; A. De Marchi, “'Cum dictum opus sit magnum?. Il documento pistoiese del $1274 \mathrm{e}$ l'allestimento trionfale dei tramezzi in Umbria e Toscana fra Due e Trecento ", dans A.C.Quintavalle (dir.) Medioevo : immagine e memoria, atti del convegno di Parma (23-28 septembre 2008), Milano, 2009, p. 603-621 ; D. Cooper, «Access all areas ? Spatial divides in the mendicant churches of late medieval Tuscany ", dans F. Andrews (éd.), Ritual and space in Middle Ages, proceedings of the 2009 Harlaxton symposium, Donington, 2011, p. 90-107.

40. J. Cannon, op. cit. note 25.

41. A. D’Amato, «S. Domenico e i primi frati a Bologna ", dans S. Gelichi et R. Merlo (dir.), Archeologia medievale a Bologna. Gli scavi nel convento di San Domenico, Bologna, 1987, p. 91-97. Sur l'évolution de l'attitude des Dominicains vis-à-vis du culte des saints, en particulier de leur fondateur, et du rôle de la papauté dans ce processus, voir A. Vauchez, $L a$ sainteté en Occident aux derniers siècles du Moyen Âge (1198-1431), Rome, 1981, p.138-141.

42. B.M. Reichert (éd.), Litterae encyclicae magistrorum generalium ordinis Praedicatorum ab anno 1233 ad annum 1376, Romae, 1900, p. 1. Traduction de l'auteur : « Gisait, de fait, un trésor caché, sans aucune utilité, de sorte que les bénéfices furent enlevés du haut du ciel par le dispensateur des vertus ; car l'équité de la justice exigeait d'enlever la grâce à ceux qui s'efforçaient de cacher la grâce et la gloire de Dieu. "

43. E. Napione, «La propaganda artistica domenicana : committenze e iconografie di un papa da inventare ", dans M. Benedetti (dir.), Benedetto XI, frate predicatore e papa, Milano, 2007, p. 147-188, p. 155.

44. J. De Backer (éd.), " De vita prima et miraculis Beati Benedicti papae XI auctore Bernardo Guidonis", in Analecta Bollandiana, $\mathrm{n}^{\circ} 19,1900$, p. 14-20. 
Conservateur général du patrimoine honoraire

\section{Françoise BouDON}

Ingénieur de recherches honoraire, CNRS

Isabelle CHAVE

Conservateur en chef du patrimoine, Archives nationales

\section{Alexandre CojAnNoT}

Conservateur du patrimoine, Archives diplomatiques

Thomas CoOMAns

Professeur, université de K.V. Leuven

Thierry CRÉPIN-LEBLOND

Conservateur général du patrimoine, directeur du musée d'Écouen

Vincent DrogueT

Conservateur en chef du patrimoine, château de Fontainebleau

Nicolas FAUCHERRE

Professeur, université d'Aix-Marseille

Pierre GarRigou Grandchamp

Général de corps d'armée (Armée de terre), docteur en Histoire de l'art et archéologie

\section{Étienne HAMON}

Professeur, université de Picardie-Jules Verne

François HEBER-SUFFRIN

Maître de conférences honoraire, université de Nanterre

Paris ouest-La Défense

Dominique Hervier

Conservateur général du patrimoine honoraire

Bertrand JESTAZ

Directeur d'études à l'École pratique des Hautes Études

\section{Claudine LAUTIER}

Chercheur honoraire, CNRS

\section{Emmanuel LURIN}

Maître de conférences, université de Paris IV-Sorbonne

\section{Jean MESQUI}

Ingénieur général des Ponts-et-Chaussées, docteur en Histoire de l'art et archéologie

Jacques Moulin

Architecte en chef des Monuments historiques

Philippe Plagnieux

Professeur, université de Besançon

Éliane Vergnolle

Professeur honoraire, université de Besançon

Directeur des publications

Rédacteur en chef

Marie-Paule ARNAULd

Éliane Vergnolle

\section{Actualité \\ Chronique \\ Bibliographie}

\section{Pierre Garrigou Grandchamp}

Dominique Hervier

Françoise BoudoN

Secrétaire de rédaction

\section{Nathalie LEBLOND}

Infographie et P.A.O.

David Leboulanger

\section{Maquette graphique L'ARCHITECTURE GRAPHIQUE}

Les articles pour publication, les livres et articles pour recension

doivent être adressés à la Société Française d'Archéologie,

5, rue Quinault, 75015 Paris

E-mail:sfa.sfa@wanadoo.fr 


$\begin{aligned} \text { Comité scientifique } & \text { Jean-Pierre BABELON } \\ & \text { Françoise BERCÉ } \\ & \text { Gabrielle DEMIANS D’ARCHIMBAUD } \\ & \text { Peter KURMANN } \\ & \text { Willibald SAUERLÄNDER } \\ & \text { Neil STRATFORD }\end{aligned}$

COMITÉ D'HONNEUR

GuY BARRUOL

Georges CosTA

Charles Duguet

Alain ERLANDE-BRANDENBURG

Michel JANTZEN

Jean-Claude RochetTE

\author{
BUREAU \\ Président Marie-Paule ARNAuld \\ Vice-Présidents Éliane Vergnolle, Bertrand JestaZ \\ Secrétaire Général Isabelle CHAVE \\ Secrétaires Généraux adjoints Philippe Dubost, Françoise Hamon \\ Trésorier Marc DE VLIEGER \\ Trésorier adjoint Bruno CHAUFFERT-YvaRT \\ Chargé de l'organisation des Congrès JEAN-Philippe RoebBen
}

\title{
CONSEIL D'ADMINISTRATION
}

Marie-Paule Arnauld, Jean-Pierre BAbelon, Françoise BerCÉ, Jean-Louis Biget, Françoise Boudon, Quitterie CAZES, Jean Chapelot, Albert Châtelet, Monique Chatenet, Bruno Chauffert-Yvart, Isabelle Chave, Philippe Contamine, Thierry Crépin-Leblond, Marc De Vlieger, Frédéric Didier, Vincent Droguet, Philippe Dubost, Yves Esquieu, Nicolas Faucherre, Bernard Fonquernie, Pierre Garrigou Grandchamp, Jean Guillaume, Françoise Hamon, Dominique Hervier, Bertrand Jestaz, Bernard Joly, Vincent Juhel, Jean-François Lagneau, Jean MesQui, Claude Mignot, Jacques Moulin, Jean-Marie Pérouse de Montclos, Philippe Plagnieux, Michel Rivet, Jean-Philippe Roebben, Élisabeth TABuret-Delahaye, Éliane Vergnolle.

\section{SITE SFA}

Découvrez le nouveau site de la Société Française d'Archéologie : www.sfa-monuments.fr 